\title{
Acute liver failure secondary to hepatic infiltration: a single centre experience of 18 cases
}

\author{
D Rowbotham, J Wendon, R Williams
}

\begin{abstract}
Background-Acute liver failure (ALF) secondary to malignant infiltration of the liver is rare and is diagnosed often only after death.

Aims-To determine diagnostic factors and particular clinical patterns of illness. Methods-Review of case notes from all patients with ALF secondary to hepatic infiltration admitted to this unit over an 18 year period (1978-1995).
\end{abstract}

Results-From a total of 4020 admissions, 18 patients were identified with ALF attributable to hepatic infiltration. Mean age was 40.7 years. Aetiology was nonHodgkin's lymphoma in nine patients, Hodgkin's disease in three, infiltrative metastatic carcinoma in four, and haemophagocytosis with no precipitant cause in two cases. Prodromal symptoms were non-specific, but occurred at least two to four weeks before onset of ALF, making the presence of such symptoms of value in differential diagnosis of the cause of ALF. Clinical examination and investigations were unhelpful in distinguishing these cases from more usual causes of ALF. Usually, the clinical course was of rapid deterioration and death from multiorgan failure, and only one patient survived. Diagnosis was made during life in 15 patients. Histology showed evidence of widespread hepatocellular necrosis, with diffuse infiltration by tumour cells rather than focal cellular aggregation.

Conclusions-Only with accurate histological diagnosis from liver biopsy and institution of specific therapy early in the management of such patients will the best chance of recovery be achieved. In every case of ALF with prodromal symptoms or abnormal imaging, hepatic histology should be obtained by liver biopsy as soon as possible to diagnose infiltrative hepatic disease.

(Gut 1998;42:576-580)

Studies, King's College

Hospital,

London SE5 9RS, UK

D Rowbotham

J Wendon

Keywords: acute liver failure; hepatic infiltration; diagnosis; histology; prognosis

Institute of

Hepatology, University

College London

Medical School,

London, UK

$\mathrm{R}$ Williams

Correspondence to:

Dr D Rowbotham.

Accepted for publication 31 October 1997 hepatocellular function is unusual until the minal deterioration, ${ }^{2}$ although there have been a number of case reports describing the occurinfiltration of the liver by malignant cell popurence of acute liver failure (ALF) secondary to lations as a presenting feature of the illness. ${ }^{3-19}$ Among these, haematological malignancies are the most common underlying aetiology, including Hodgkin's disease, ${ }^{3-5}$ non-Hodgkin's lymphoma, ${ }^{6-10}$ malignant histiocytosis,${ }^{11-13}$ and the leukaemias (acute and chronic). ${ }^{14-16}$ Other infiltrative metastatic malignancies that rarely can cause ALF include adenocarcinomas, melanoma, and anaplastic tumours from various primary sites. ${ }^{17-23}$ Making a diagnosis of hepatic infiltration in patients presenting with ALF in such cases is difficult and requires a high degree of suspicion if evidence of the underlying primary disease is absent, as is usually the case. The finding of lymphadenopathy along with jaundice points to hepatic lymphomatous infiltration, ${ }^{24}$ but more commonly the presenting symptoms and signs are indistinguishable from primary hepatic causes and the diagnosis often is not made until postmortem. Death usually is as a direct consequence of the ALF, rather than due to the underlying malignancy. However, early diagnosis and specific treatment of such cases, particularly with the infiltrating haematological malignancies, can lead to resolution of the hepatic failure in response to the destruction of the infiltrating cell population. ${ }^{1015}$

Prompted by two recent cases of ALF secondary to infiltrative hepatic disease, we have reviewed our experience with this rare clinical situation in adults in a single centre over the past 18 years to determine diagnostic factors and particular clinical patterns of illness.

Methods and results

Over the period 1978-1995, 18 patients with ALF shown to be attributable directly to hepatic infiltration (either during life or at autopsy) were transferred to the Liver Failure Unit at King's College Hospital. ALF was defined as the syndrome of hepatic encephalopathy and other manifestations of liver cell failure in the absence of pre-existing liver disease and fulfilling criteria as defined by O'Grady et al. $^{25}$

The 18 patients comprised $0.44 \%$ of the 4020 patients admitted to the Liver Failure Unit over this time period. Sex distribution of the 18 cases was equal and age range was 16-73 years (mean 40.7 years; median 38 years). The underlying aetiology was nonHodgkin's lymphoma (NHL) in nine patients, Hodgkin's disease (HD) in three, metastatic carcinoma (CA) in four, and haemophagocytic syndrome with no clear precipitant cause in two. One patient presented initially with haemophagocytic syndrome, but was diagnosed 
subsequently at postmortem to have NHL. Mean time from appearance of jaundice to admission to this unit was four days (range 2-14). The majority of patients fulfilled the criteria for hyperacute liver failure (development of encephalopathy within seven days of onset of jaundice), with a minority coming within the category of acute liver failure (jaundice to encephalopathy time of 8-28 days). ${ }^{25}$

Median time to transfer after admission was six days and median time to diagnosis was eight days after admission to hospital. Diagnosis of the underlying disease process was established by the referral hospital in only four patients. Overall diagnosis during life was achieved in 15 patients on the basis of histological analysis of tissue from liver biopsy (seven patients), bone marrow biopsy (five), lymph node biopsy (two), and by appearances seen at ultrasound scanning (one). In the remaining three patients, a postmortem diagnosis was made from histological analysis of hepatic tissue in two patients and lymph node tissue in one. In all, 19 biopsies were performed in 16 patients and before a diagnosis could be made, three patients underwent biopsy of more than one tissue (two bone marrow/liver combinations and one bone marrow/lymph node). Bone marrow samples were obtained from nine patients, lymph node tissue from two, and hepatic tissue from eight. Liver biopsies were performed using a percutaneous needle approach in five patients, the transjugular route in one, and at laparotomy in two.

There was a history of previous therapy for the same disease in 1/8 NHL patients and in 2/3 HD cases. Two patients who developed ALF secondary to NHL were receiving immunosuppressive therapy at the time of presentation with ALF. One of these was on a combination of prednisolone, azathioprine, and cyclosporin $\mathrm{A}$ as routine immunosuppression following cardiac transplantation two years earlier. This patient showed a significant IgM titre to Epstein-Barr virus, but was known to have suffered very recently from infectious mononucleosis. Another patient with ALF secondary to NHL was taking a combination of prednisolone and azathioprine as treatment for an autoimmune haemolytic anaemia diagnosed two years previously.

Prodromal symptoms were present usually for at least two to four weeks before development of ALF, although the range was wide: five days to two months (mean 28 days). No patient developed ALF without prodromal symptoms. The usual prodromal symptoms were nonspecific, comprising malaise (in $50 \%$ of cases), weight loss $(39 \%)$, right upper quadrant abdominal pain (39\%), and fever (33\%). Jaundice appeared later in the disease course and heralded the onset of ALF. Mean time from appearance of jaundice to hospital admission was four days (range 2-14 days).

Physical signs on admission were of help in diagnosis only in respect to the NHL subgroup, in whom palpable lymphadenopathy was present in 5/9 cases. No other patient had clinical lymphadenopathy, although two patients (one $\mathrm{HD}$ and one $\mathrm{CA}$ ) were found to have intra-abdominal lymphadenopathy on computed tomography (CT) imaging. The depth of jaundice on presentation was variable, but all patients were icteric. Hepatomegaly was more pronounced than in other causes of ALF, with the liver palpable 2-5 fingerbreadths below the costal margin and with a firm edge. It was present with equivalent frequency in patients from all diagnostic subgroups. Splenomegaly, by contrast, was noted only in the NHL and HD subgroups. Postmortem examination of the enlarged spleens revealed infiltration by tumour cells as the cause of the splenomegaly in all cases. One half of patients were febrile at presentation despite only one third reporting fever as a presenting symptom.

Diagnosis in the three patients who presented with haemophagocytic syndrome was based on histological analysis of bone marrow aspirate and trephine biopsy. One patient was discovered subsequently to have NHL, but in the remaining two no precipitant cause could be established despite extensive antemortem and postmortem investigation. Both of these patients were young women aged 30-40 years. One of these patients gave a history of three weeks of cough and sore throat for which she had been given a seven day course of oral amoxycillin, but all bacteriological and viral markers were negative. The other patient had no preceding symptoms and was negative for the whole range of viral and bacteriological investigations. Neither patient had taken any other drugs, either prescribed or self medicated, nor were they pregnant.

As most patients were transferred early on in the course of ALF, notable coagulopathy was not a feature on admission (INR range 1.0-6.1, median 3.02), but progressed rapidly following admission (median maximum value of INR 6.1). Thrombocytopenia was a feature in all patients (platelet count range 10-326 $\times 10^{9} / 1$, median 60 ), with the majority also displaying a mild anaemia (haemoglobin range 6.3-13.6 $\mathrm{g} / \mathrm{dl}$, median 10.5) and an increased peripheral blood leucocyte count (range 0.9-26.0 × 10 $/ 1$, median 7.4). None of these results were of value in discriminating these patients from those with ALF from other causes. Electrolyte disturbances were common, especially hyponatraemia (sodium range $117-146 \mathrm{mmol} / \mathrm{l}$, median 129; potassium range $2.9-6.8 \mathrm{mmol} / \mathrm{l}$, median 4.1) along with evidence of renal impairment (urea range $4.1-50 \mathrm{mmol} / \mathrm{l}$, median 20.1; creatinine range 51-645 $\mu \mathrm{mol} / 1$, median 119). Liver function tests showed impairment of synthetic function with low serum albumin (range 18-39 g/l, median 29) in addition to evidence of severe hepatocellular injury with high serum aminotransferase levels (aspartate aminotransferase range 79-2180 IU/1, median 358; $\gamma$ glutamyl transpeptidase range 78-768 IU/1, median 218), although serum aminotransferase were not as high as has been found in other causes of ALF. The level of jaundice on admission was variable in the majority of patients (bilirubin median 187 $\mu \mathrm{mol} / \mathrm{l})$, although a minority were deeply jaundiced (bilirubin $>100 \mu \mathrm{mol} / 1$ in 14 patients and $>350 \mu \mathrm{mol} / 1$ in three). Three patients with 
infiltrative lymphoma showed predominantly cholestatic jaundice with gross elevation of alkaline phosphatase values (>1500 IU/1), but most patients showed only modest elevation (range 121-4620 IU/1, median 522).

Radiological imaging, by ultrasound scanning in 16 and CT in seven patients, although not providing a definitive diagnosis, was of value in revealing the presence of intraabdominal lymphadenopathy or confirming hepatosplenomegaly in eight patients. Increased reflectivity and abnormal echotexture of the hepatic parenchyma, typical of infiltrative hepatic disease, was recorded in eight patients.

\section{Clinical course and outcome}

The clinical course following admission, with the exception of one patient, was of rapid deterioration and death. Overall mortality was $94 \%$, the only survivor being from the NHL subgroup. Median time to death following admission was six days (range 1-51 days). Death was caused in all patients by multiorgan failure due to combinations of cardiovascular collapse, acute lung injury, and renal failure in addition to hepatic failure. In the 24 hours before death, all patients had clinical evidence of sepsis (fever, peripheral blood leucocytosis, urine microscopy, or radiological changes of pneumonia).

The progressive clinical deterioration and high mortality occurred despite the prompt administration of specific treatment (chemotherapy, corticosteroids, and/or immunoglobulin) to 11 of the 15 patients in whom an antemortem diagnosis had been established. Two patients with NHL were considered too unwell to receive any form of chemotherapy and two patients found to have infiltrative metastatic carcinoma were treated initially with chemotherapy on the clinical suspicion of underlying lymphoproliferative disease. The haemophagocytic syndromes with no precipitant cause were treated empirically with corticosteroids, intravenous acyclovir, and chemotherapy (etoposide in one patient, methotrexate in the other). Their condition improved initially in the first few weeks, but in both cases the haemophagocytic syndrome relapsed leading to rapid deterioration and death within three and six weeks of admission respectively.

The one survivor in the series was a 27 year old man with NHL who had presented with a 17 day history of diarrhoea, vomiting, fever, and weight loss and five days of increasing jaundice. He had palpable hepatosplenomegaly and CT imaging revealed retroperitoneal and para-aortic lymphadenopathy. Rapid deterioration occurred with coagulopathy (INR 3.9), a rising alkaline phosphatase (>1000 IU/1), and encephalopathy (grade 3 ). A percutaneous liver biopsy, performed by the referring hospital four days after presentation, confirmed a diagnosis of infiltrative NHL. He was given intravenous cyclophosphamide the next day and transferred to our unit. Combination intravenous chemotherapy (doxorubicin, vincristine, corticosteroids, and immunoglobulin) was instituted immediately, with a second course of intravenous chemotherapy (cyclophosphamide, vincristine, doxorubicin, and oral steroids) four weeks later. He subsequently made a full recovery.

Histological diagnosis was made from the specific features of the infiltrating cell population. Whether on needle biopsy, or at postmortem, the infiltrating cell populations were spread diffusely throughout liver tissue with no evidence of localised or focal cellular aggregation. There was no association between the extent or pattern of cellular infiltration and underlying aetiology. Large areas of liver showed evidence of necrosis, such necrotic change ranging from subacute to severe. Interestingly, necrosis was not confined to infiltrated areas of hepatic tissue, with non-infiltrated tissue also showing evidence of necrosis.

\section{Discussion}

This series, limited to adults only, adds considerably to the reported experience of ALF secondary to hepatic infiltration. The findings confirm previous reports with haematological malignancies being implicated in the majority of cases. Of these, NHL is consistently the commonest cause. Although liver involvement is found in $16-22 \%$ of cases of untreated NHL, ${ }^{26-28}$ such hepatic disease is most often asymptomatic. It can be associated, on occasion, with hepatomegaly and derangement in liver function tests, especially a rise in alkaline phosphatase, ${ }^{24}{ }^{26-28}$ but without liver failure. In none of the present patients, however, was there evidence of such hepatic involvement antedating presentation with ALF. In cases of ALF secondary to lymphoproliferative disease, a history of previous treatment for the same disease was apparent in over one quarter of cases. This suggests that recurrence of a previously treated lymphoma may predispose to a subsequent preferential aggressive invasion of the liver, although by what mechanism is unclear.

One patient, found to have underlying NHL, presented with ALF secondary to haemophagocytosis. Such a condition does not occur as a primary event. Potential precipitants of acute haemophagocytic syndrome include viral infection, ${ }^{29-34}$ bacterial or fungal infection, ${ }^{35-38}$ metabolic disturbance, ${ }^{39}$ inflammatory arthropathy, ${ }^{40}$ and pregnancy ${ }^{41}$ in addition to leukaemias and lymphoproliferative disease. ${ }^{42-44}$ The possibility of lymphoma as a cause of the acute haemophagocytic syndrome in the two patients without a clear precipitant aetiology seems very unlikely, since investigation during life and postmortem examination failed to provide evidence of such disease.

It is important, both in terms of early diagnosis and prompt initiation of treatment, to recognise symptoms associated with the underlying disease from those due to the development of ALF. Disease related prodromal symptoms were usually present at least two to four weeks before development of ALF. No patient developed ALF without prodromal symptoms. This is of diagnostic help in distinguishing these cases from ALF due to other causes. The depth of jaundice on admission 
was lower in this series than with other causes of $\mathrm{ALF}^{19}$ although this finding was variable and unhelpful in distinguishing these cases from ALF due to other causes.

Palpable lymphadenopathy, which may have led to suspicion of haematological malignancy, was present in surprisingly few cases. The apparent predilection of the tumours in these patients to infiltrate the liver, often in isolation, in preference to the peripheral lymphatic system, raises the possibility of a phenotypic lymphomatous subtype with selective organ invasion. In view of the diagnostic implications in such patients, any uncertainty regarding the underlying aetiology in a patient with ALF must be clarified by liver biopsy (or bone marrow biopsy) in order to achieve an accurate diagnosis. This is of particular importance in relation to the possible use of orthotopic liver transplantation for ALF. Secondary malignant infiltration of the liver is an absolute contraindication to orthotopic liver transplantation.

Mortality in this series is in agreement with previously published reports. The sole survivor from our series had a number of favourable factors including his young age and a diagnosis of high grade NHL, a tumour likely to exhibit a good response to chemotherapeutic intervention. Nevertheless, it was the attainment of a definitive histological diagnosis by percutaneous liver biopsy that allowed prompt administration of specific chemotherapy. A histological diagnosis was obtained from bone marrow or lymph node biopsy as often as from liver biopsy, reflecting the preponderance of haematological malignancies in this series. Although the risks of percutaneous biopsy are increased in such cases due to concurrent coagulopathy and/or thrombocytopenia, with appropriate fresh frozen plasma and/or platelet transfusion cover, there was no additional morbidity or mortality as a result of the biopsies carried out in this series.

Within the spectrum of malignant disease, the liver is the most common site for metastatic tumour deposits $(36 \%)$, the incidence being highest $(48 \%)$ for tumours arising within the portal venous drainage area. ${ }^{1}$ Metastatic spread to the liver occurs usually as discrete tumour deposits, either single or multiple, or less commonly as hepatic invasion by sheets of malignant cells in a more diffuse pattern. Various mechanisms have been suggested to explain the association of hepatic infiltration and ALF including tumour infiltration of the biliary tree, hepatic vasculature, and hepatic parenchyma. Invasion of the extrahepatic biliary system leads to obstructive jaundice rather than ALF, but infiltration of small intrahepatic bile ducts may result in extensive cholangitis, duct necrosis, and ALF. ${ }^{45}$ Obstruction of hepatic venules by tumour may result in hepatocyte ischaemic injury and necrosis, ${ }^{15}$ or massive sinusoidal infiltration by malignant cells is likely to cause sudden ischaemia and subsequent hepatocellular necrosis. ${ }^{18}$ Alternatively, rapid replacement of vast areas of hepatic parenchyma by malignant cells may lead to a critical mass of hepatocyte destruction and subsequent ALF. ${ }^{1517} 19$
The potential role of cytokines in ALF associated with malignant disease, but especially in lymphomas, has been the cause of much recent interest. It has been suggested that massive cytokine release from lymphomatous cells may cause interlobular bile duct destruction and portal fibrosis, the so called vanishing bile duct syndrome. ${ }^{46-49}$ Cytokines may damage bile ducts directly or may result in the recruitment of other immunological effector cells, leading ultimately to bile duct destruction. This might account for the observed disparity between relatively minor lymphomatous cell loads and the very severe cholestasis that occurs in reported cases of idiopathic Hodgkin's associated cholestasis. ${ }^{46}{ }^{50}$ Interleukin 2, which is one of the cytokines released from lymphoma cells, for example, has been shown to induce hepatocyte toxicity by activating Kupffer cells with release of further cytokines (such as tissue necrosis factor) leading to activation of circulating leucocytes and hepatic sinusoidal endothelial cells. The subsequent leucocyte and platelet adhesion to sinusoidal endothelium physically impedes the sinusoidal microcirculation resulting in microscopic hepatic ischaemia. ${ }^{51}$ Such mechanisms, if occurring diffusely, may lead to widespread hepatocyte dysfunction and ALF. It is possible that massive release of cytokines from malignant cell populations anatomically distinct from the liver may lead to ALF in a similar way, since occasional instances of fulminant hepatic failure have been described in non-metastatic renal cell carcinoma. ${ }^{52}$

It has been shown by using genetically tagged cells that metastatic cell subpopulations can outgrow their non-metastatic counterparts within the primary tumour, ${ }^{53}$ suggesting that the metastatic potential of a primary tumour may increase during the course of its growth. Subsets of cells within a tumour may show a predilection for certain metastatic sites, but the relevance of this to human cancers is unproven. ${ }^{54}$ Nevertheless, the possibility of subgroups of cells within a cancer that express varying degrees of aggression or malignancy may be an important factor in determining not only the site of metastasis, but possibly also the occurrence of ALF in rare cases. Recurrence of a previously treated lymphoma could also be explained on this basis. In this series, a history of previous treatment for the same disease was present in over one quarter of patients with lymphoma. This fact raises the possibility that chemotherapy may change or enhance the malignant behaviour of such tumours with recurrent tumour cell subpopulations possessing enhanced malignant properties which preferentially target and invade the liver.

This work was presented in abstract form (Acute liver failure due to hepatic infiltration; D S Rowbotham, J A Wendon, $R$ Williams) at the Liver Intensive Care Group of Europe, ninth meeting in Helsinki, 1996.

\footnotetext{
1 Willis RA. The spread of tumours in the human body. London:

2 Cello JP, Grendell JH. The liver in systemic conditions. In: Zakim D, Boyer TD, eds. Hepatology. A textbook of liver disease. Philadelphia: WB Saunders, 1990:1423-5.
} 
3 Fairbank WHD. Three atypical cases of Hodgkin's disease presenting with liver failure. Can Med Assoc F 1953;69:315 17.

4 Trewby PN, Portmann B, Brinkley DM, et al. Liver disease as presenting manifestation of Hodgkin's disease. $Q \mathcal{F ~ M e d}$ 1979;48:137-50.

5 Lefkowitch JH, Falkow S, Whitlock RT. Hepatic Hodgkin's disease simulating cholestatic hepatitis with liver failure. Arch Pathol Lab Med 1985;109:424-6.

6 Braude S, Gimson AE, Portmann B, et al. Fulminant hepatic failure in non-Hodgkin's lymphoma. Postgrad Med 7 1982;58:301-4.

7 Ghosh P, Fox IJ, Rader AM, et al. Fulminant hepatic failure as the initial manifestation of non-Hodgkin's lymphoma. Am f Gastroenterol 1995;90:2207-9.

8 Woolf GM, Petrovic LM, Rojter SE, et al. Acute liver failure due to lymphoma. A diagnostic concern when considering liver transplantation. Dig Dis Sci 1994;39:1351-8.

9 Gargot D, Maitre F, Causse X, et al. Primary liver non-Hodgkin's lymphoma presenting as fulminant hepatic disease. Eur f Gastroenterol Hepatol 1994;6:843-6.

10 Salo J, Nomdedeu B, Bruguera $M$, et al. Acute liver failure due to non-Hodgkin's lymphoma. Am $\mathcal{f}$ Gastroenterol 1993;

11 Colby TV, LaBrecque DR. Lymphoreticular malignancy presenting as fulminant hepatic disease. Gastroenterology 1982;82:339-45.

12 Crotty CP, Winkelmann RK. Cytophagic histiocytic panniculitis with fever, cytopenia, liver failure and termina haemorrhagic diathesis. $\mathcal{f}$ Am Acad Dermatol 1981;4:18194.

13 Beaugrand M, Trinchet JC, Callard P, et al. Malignant histiocytosis presenting as a fulminant hepatic disease [letter] Gastroenterology 1983;84:447-8.

14 Hess CE, Joyce RA. Acute myeloblastic leukaemia presenting as progressive hepatic failure. South Med F 1981;74: ing as prog

15 Zafrani ES, Leclercq B, Vernant JP, et al. Massive blastic infiltration of the liver: a cause of fulminant hepatic failure. infiltration of the liver: a cause

16 Greer JP, Cousar JB, Miller RF, et al. $\mathrm{T}^{+}$(helper phenotype) chronic lymphocytic leukaemia: a case report with liver failure and literature review. Med Pediatr Oncol 1988;16:356-62

17 Eras P, Sherlock P. Hepatic coma secondary to metastatic liver disease. Ann Intern Med 1971;74:581-3.

18 Harrison HB, Middleton HM, Crosby JH, et al. Fulminan hepatic failure: an unusual presentation of metastatic liver disease. Gastroenterology 1981;80:820-5.

19 Myszor MF, Record CO. Primary and secondary malignant disease of the liver and fulminant hepatic failure. $\mathcal{F}$ Clin Gastroenterol 1990;12:441-6.

20 Krauss EA, Ludwig PW, Sumner HW. Metastatic carcinoma presenting as fulminant hepatic failure. Am $\mathcal{f}$ Gastroenterol 1979;72:651-4.

21 Nouel O, Bernuau J, Lebrec D, et al. Severe hepatic failure and portal hypertension due to metastatic carcinoma of the liver. Gastroenterol Clin Biol 1979;3:135-7.

22 Bouloux PM, Scott RJ, Goligher JE, et al. Fulminant hepatic failure secondary to diffuse liver infiltration by melanoma. failure secondary to diffuse 1 .
$\exists R$ Soc Med 1986;79:302-3.

23 Stein AM, Fawaz K, Tabrizi A, et al. Multifocal malignant haemangioendothelioma presenting as acute hepatitis. A clinicopathologic study. Am f Gastroenterol 1977;67:370-4

24 Biemer JJ. Hepatic manifestations of lymphomas. Ann Clin Lab Sci 1984;14:252-60.

25 O'Grady JG, Schalm SW, Williams R. Acute liver failure: redefining the syndromes. Lancet 1993;342:273-5.

26 Roth A, Kolaric K, Dominis M. Histologic and cytologic liver changes in 120 patients with malignant lymphomas. Tumori 1978;64:45-53.

$27 \mathrm{Kim} \mathrm{H}$, Dorfman RF. Morphological studies of 84 untreated patients subjected to laparotomy for the staging of non-Hodgkin's lymphoma. Cancer 1974;33:657-74

28 Fergusson DJ, Allen LW, Griem ML, et al. Surgical experience with staging laparotomy in 125 patients with experience with staging laparotomy in 125 p
lymphoma. Arch Intern Med 1973;131:356-61.

29 Sasadeusz J, Buchanan M, Speed B. Reactive haemophagocytic syndrome in human immunodeficiency virus infection. F Infect 1990;20:65-8.
30 Huang LM, Lee CY, Lin KH, et al. Human herpesvirus-6 associated with fatal haemophagocytic syndrome [letter]. Lancet 1990;336:60-1.

31 Ohshima K, Kikuchi M, Eguchi F, et al. Virus-associated haemophagocytic syndrome with Epstein-Barr virus infection. Virchows Arch A 1991;419:519-22.

32 Ramanathan M, Duraisamy G. Haemophagocytosis in dengue haemorrhagic fever: a case report. Ann Acad Med Singapore 1991;20:803-4.

33 Watanabe $M$, Shimamoto Y, Yamaguchi $M$, et al. Viralassociated haemophagocytosis and elevated serum TNFalpha with parvovirus-B19-related pancytopenia in patients with hereditary spherocytosis. Clin Lab Haematol 1994;16: 179-82.

34 Kondo H, Date Y. Effects of simultaneous rhG-CSF and methylprednisolone "pulse" therapy on hepatitis A virusassociated haemophagocytic syndrome. Eur f Haematol 1995;54:271-3

35 Bhatia VN, Parmar U, Subramanian C, et al. Virulence, haemolysis pattern and haemophagocytosis index of Entamoeba histolytica. Indian f Med Res 1982;76:545-51.

36 Niggli F, Halle F, Seger RA. Immunologic complications of visceral leishmaniasis. Monatsschrift Kinderheilkunde 1991; 139:772-4.

37 Ramanathan M, Karim N. Haemophagocytosis in typhoid fever. Med f Malaysia 1993;48:240-3.

38 Quiquandon I, Plantier I, Hatron PY, et al. Tuberculosis associated haemophagocytic syndrome: two cases with a favourable outcome. Nouv Rev Fr Hematol 1995;37:149-

39 Mandel H, Gozal D, Aizin A, et al. Haemophagocytosis in hereditary fructose intolerance: a diagnostic dilemma. $\mathcal{F}$ Inherit Metab Dis 1990;13:267-9.

40 Neve P, Decaux G. Still's disease in adults. Rev Med Brux 1991;12:399-401.

41 Gill DS, Spencer A, Cobcroft RG. High-dose gamma globulin therapy in the reactive haemophagocytic syndrome. Br f Haematol 1994;88:204-6.

42 Stark R, Manoharan A. Haemophagocytic syndrome complicating acute

43 Wong $\mathrm{KF}$, Chan JK, Ng CS, et al. Erythroleukaemiaassociated haemophagocytic syndrome. Clin Lab Haematol 1991;13:383-6.

44 Prussia PR, Mansoor GA, Edwards C, et al. Haemophagocytic syndrome. W Indian Med $\mathcal{F} 1991 ; 40$

45 Dich NH, Goodman ZD, Klein MA. Hepatic involvement in Hodgkin's disease: clues to histologic diagnosis. Cancer 1989;64:2121-6.

46 Hubscher SG, Lumley MA, Elias E. Vanishing bile duct syndrome: a possible mechanism for intrahepatic cholestasis in Hodgkin's lymphoma. Hepatology 1993;17:70-7.

47 Hsu SM, Xie SS, Hsu PL, et al. Interleukin-6, but not interleukin-4, is expressed by Reed-Sternberg cells in Hodgkin's disease with or without histologic features of Castleman's disease. Am f Pathol 1992;141:129-38.

48 Reinhold U, Abken H, Kukel S. Tumor infiltrating lymphocytes isolated from a $\mathrm{Ki}-1$ positive large cell lymphoma of the skin. Phenotypic characterization and analysis of cytokine secretion. Cancer 1991;68:2155-60.

49 Woolf GM, Vierling JM. Disappearing intrahepatic bile ducts: the syndromes and their mechanisms. Semin Liver ducts: the syndromes

50 Perera DR, Greene ML, Fenster LF. Cholestasis associated with extrabiliary Hodgkin's disease. Gastroenterology 1974; 67:680-5.

51 Nakagawa K, Miller FN, Sims DE, et al. Mechanisms of interleukin-2-induced hepatic toxicity. Cancer Res 1996;56: $507-10$

52 Fang JW, Lau JY, Wu PC, et al. Fulminant hepatic failure in non metastatic renal cell carcinoma. Dig Dis Sci 1992;37: 474-7.

53 Kerbel RS, Waghorne C, Korczac B, et al. Clonal dominance of primary tumours by metastatic cells: genetic analysis and biological implications. Cancer Surv 1988;7: 597-629.

54 McMillan TJ, Hart IR. Can cancer chemotherapy enhance the malignant behaviour of tumours? Cancer and Metastasis Reviews 1987;6:505-20. 